\title{
Phase modulation of the bucket stops bunch oscillations at the Fermilab Tevatron
}

\author{
C. Y. Tan and A. Burov \\ Fermi National Accelerator Laboratory, P.O. Box 500, Batavia, Illinois 60510-5011, USA
}

(Received 18 April 2011; published 23 April 2012)

\begin{abstract}
Bunches in the Tevatron are known to exhibit longitudinal oscillations which persist indefinitely. These oscillations are colloquially called "dancing bunches." Although the dancing proton bunches do not cause single bunch emittance growth or beam loss at injection, they lead to bunch lengthening at collisions. In Tevatron operations, a longitudinal damper has been built which stops this dance and damps out coupled bunch modes. Recent theoretical work predicts that the dance can also be stopped by an appropriate change in the bunch distribution. This paper describes the Tevatron experiments which support this theory.
\end{abstract}

DOI: 10.1103/PhysRevSTAB.15.044401

PACS numbers: 29.27.Bd, 29.27.Fh

\section{INTRODUCTION}

Since the start of Run II, the proton bunches in the Tevatron have been observed to have longitudinal oscillations which persist indefinitely. The initiators of these oscillations for coalesced [1] bunches come from the coalescing process (see Sec. III A 2) and possibly also from injection errors [2]. The reason for the persistence of these oscillations has been traced to the loss of Landau damping (LLD) caused by the inductive impedance of the Tevatron [3]; these oscillations are colloquially called "dancing bunches." At the injection energy of $150 \mathrm{GeV}$, these oscillations do not seem to cause any emittance growth or any beam loss. But at the flattop energy of $980 \mathrm{GeV}$, they lead to an effective bunch length growth which reduces luminosity. A longitudinal damper system has been built which damps out the dance [4].

Recent theoretical work has predicted that the dance can also be stopped by flattening out its phase space distribution at low synchrotron frequencies [5,6]. In particular, this flattening can be achieved by modulating the rf phase at the synchrotron frequency of the low amplitude particles [7]. The goal of this paper is to demonstrate experimentally that the dance can be stopped by changing the beam distribution appropriately.

\section{THEORY}

The Boltzmann-Jeans-Vlasov equation [8] is conventionally used to describe longitudinal motion of bunched beams. This equation has a continuous spectrum and, possibly, a discrete one [9,10]. The discrete van Kampen modes are described with regular functions and some of them do not decay. Therefore, in principle, any coupled bunch wake drives an instability when there is LLD.

Published by the American Physical Society under the terms of the Creative Commons Attribution 3.0 License. Further distribution of this work must maintain attribution to the author(s) and the published article's title, journal citation, and DOI.
However, in practice, the coupled bunch wake has to be high enough to give an observable growth rate. If the growth rate is too small, LLD results in persistent oscillations caused by initial perturbations.

For bunched beams, LLD was first discussed and estimated by Sacherer [11]. Later, his main results were rederived and discussed in more detail by other authors [12-16]. For a dipole mode, all of the approaches were actually based on the assumption that the bunch moves as a rigid body. However, recent solutions of the eigenvalue problem $[5,6]$ show that the rigid bunch approximation can lead to significant overestimation of the LLD threshold.

As it is shown in the original paper by Sacherer [11], the threshold bunch population $N_{\text {th }}$ is a strong function of the bunch length $\ell$. In particular, for an inductive wake above transition,

$$
N_{\text {th }} \propto \ell^{5} .
$$

This scaling law can be derived from the idea that Landau damping is lost whenever the incoherent tune shift $\Delta \Omega \propto$ $N Z_{\|}(c / \ell) / \ell^{2}$ exceeds the incoherent tune spread $\delta \Omega \propto \ell^{2}$, where $N$ is the bunch population and $Z_{\|}$is the longitudinal impedance at frequency $c / \ell$. For the inductive impedance, the incoherent tune shift decreases with the bunch length as $\Delta \Omega \propto \ell^{-3}$. The combined action of this decrease with increasing nonlinear tune spread $\delta \Omega \propto \ell^{2}$ results in $\ell^{5}$ in Eq. (1). This high sensitivity to bunch length indicates that approximations of the bunch profile or arbitrary assumptions about the eigenfunctions can lead to significant errors in the calculated LLD threshold because they can change the effective bunch length. For example, for a full bucket of a single-harmonic rf system with an inductive impedance above transition, the threshold relative tune shift $\Delta \Omega / \Omega$ was found to be as low as $10 \%$ for the Hofmann-Pedersen distribution, and just $\sim 1 \%$ for a model of the Tevatron coalesced bunch [5]. In terms of bunch population, the two thresholds differ by almost 2 orders of magnitude. It turns out that the onset of LLD is highly sensitive to the steepness of the distribution function at low amplitudes: the flatter the distribution, the more stable it is. This prediction 
appears to be generally correct when the bare rf synchrotron frequency monotonically decreases with amplitude and the wakefield is repulsive, i.e., the wake lowers the incoherent synchrotron frequencies. For example, space charge below transition, inductance or resistive wall above transition are all repulsive. This conclusion agrees with Ref. [15], where the LLD threshold was calculated for several distributions with the inductive impedance above and below transition. It was shown there that below transition LLD is sensitive to the edges of the distribution, while above transition, it is sensitive to the flatness of the bunch core.

As was discussed in Ref. [5], in the case of a sinusoidal rf system, any combination of inductance, wall resistivity, high order cavity modes above transition, or space charge below transition will shift the incoherent spectrum down to lower frequency and the coherent mode will emerge above it. Since the incoherent frequencies of low amplitude particles are close to the mode frequency, their weight in the mode dominates. Hence, for a single-harmonic rf system and a repulsive wake function, the discrete mode causes dipole motion of the bunch center while its tails remain still. This is the behavior of the bunches in the Tevatron [3].

\section{A. Flattening out the distribution for particles with small amplitudes}

To flatten out the bunch distribution at small amplitudes in the Tevatron, resonant phase modulation of the rf phase was suggested [7], with the idea of using anomalous diffusion within a controlled phase space area; see Refs. [17-19] and references therein.

Let it be assumed that the rf phase is modulated at a frequency $\Omega_{m}$, which is close to the synchrotron frequency $\Omega_{s}$. Let the amplitude of the modulation $\phi_{m}(t)$ adiabatically grow from zero, then stay a while at some value $\phi_{0}$, and then adiabatically decrease to zero. To prevent excitation of the tail particles and the coherent modes, the process must be adiabatic. However, even when the process is generally adiabatic, i.e., when $\left|d \phi_{m} / d t\right| \ll \Omega_{s} \phi_{0}$, the adiabaticity for some particles will be broken. Indeed, resonant rf phase modulation results in either one or two stable fixed points (SFPs) inside the bucket. In the last case, there is an inner separatrix between the two SFPs and when the modulation amplitude changes, the separatrix moves and some particles cross it. Separatrix crossing is a nonadiabatic process resulting in classical chaos and anomalous diffusion.

Thus, the phase space density can be changed only in the case of two SFPs which occur when the modulation frequency is lower than the synchrotron frequency, $\Omega_{m}<\Omega_{s}$, and the modulation amplitude is lower than its bifurcation value, $\phi_{m}<\phi_{b}=3.08 \epsilon^{3 / 2}$ with $\epsilon=1-\Omega_{m} / \Omega_{s}$. When the modulation amplitude grows from zero to its bifurcation value, and when it comes back to zero later, the irreversible change of the phase space density occurs for the phase space area with action $J \leq J_{\lim }$, where

$$
J_{\text {lim }} \approx 6 \epsilon J_{\text {bucket }},
$$

and $J_{\text {bucket }}$ is the bucket acceptance. For dimensionless variables associated with the unperturbed Hamiltonian $H(z, p)=p^{2} / 2+1-\cos z$, the acceptance $J_{\text {bucket }}=$ $8 / \pi$. The dimensionless variables are the same as in Ref. [19], page 265, with time measured in radians of the synchrotron phase. The numerical factor "6" in Eq. (2) was approximated using a numerical solution discussed below and it is about 2 times larger than the separatrix border at zero amplitude. After this adiabatic cycle, the phase space density becomes nearly constant for the entire area $J<J_{\text {lim }}$, provided that the modulation amplitude crosses its bifurcation value, i.e.,

$$
\phi_{0} \geq 3.08 \epsilon^{3 / 2} .
$$

It is worth mentioning that the adiabatically ramped modulation does not excite any coherent motion when the modulation is turned off. Thus, to make a flat phase space density within a certain action $J_{\text {lim }}$, the adiabatic $\mathrm{rf}$ phase modulation has to be applied slightly below the synchrotron frequency, $\epsilon=0.16 J_{\text {lim }} / J_{\text {bucket }}$, and its amplitude must cross the bifurcation value in Eq. (3).

A simulation of how the bunch distribution is modified with rf phase modulation has been done using the following map:

$$
\begin{aligned}
z_{n+1} & =z_{n}+p_{n} \Delta t \\
p_{n+1} & =p_{n}-\Delta t \sin \left[z_{n+1}-\phi_{m}\left(t_{n}\right) \sin (1-\epsilon) t_{n}\right] \\
t_{n+1} & =t_{n}+\Delta t,
\end{aligned}
$$

where $z_{n}$ and $p_{n}$ are the coordinate and momentum, respectively, in dimensionless units, $t_{n}$ is the time variable in radians of the synchrotron oscillation, and $\Delta t$ is its numerical step. The amplitude of the rf phase modulation $\phi_{m}(t)$ was taken to be a trapezoid similar to that shown in Fig. 4,

Here are the typical parameters used in the simulations: (i) the adiabaticity parameter $\dot{\phi}_{m} /\left(\Omega_{s} \phi_{0}\right) \sim 200$; (ii) $\Delta t=0.01$ radians which is small enough for the results to be independent of its specific value; (iii) initial phase space density is assumed to be $F(J) \propto\left(J_{\max }-J\right)^{2}$ with the emittance $J_{\max }$ set close to the bucket acceptance; and (iv) number of macroparticles $N=4 \times 10^{4}$.

The simulation results before and after phase modulation are shown in Fig. 1 for $\epsilon=0.03, \phi_{0}=0.025$, and two consecutive phase modulation cycles with $T_{\text {sim }}=600$ radians or about 90 synchrotron periods each. Each cycle time was equally divided into three parts of about 30 synchrotron periods each: a linear growth of the modulation amplitude from 0 to $\phi_{0}$, staying at $\phi_{0}$, and a linear decrease from $\phi_{0}$ back to 0 .

Clearly, the action distribution PDF [J] has successfully flattened out and there is even a little divot that is less 

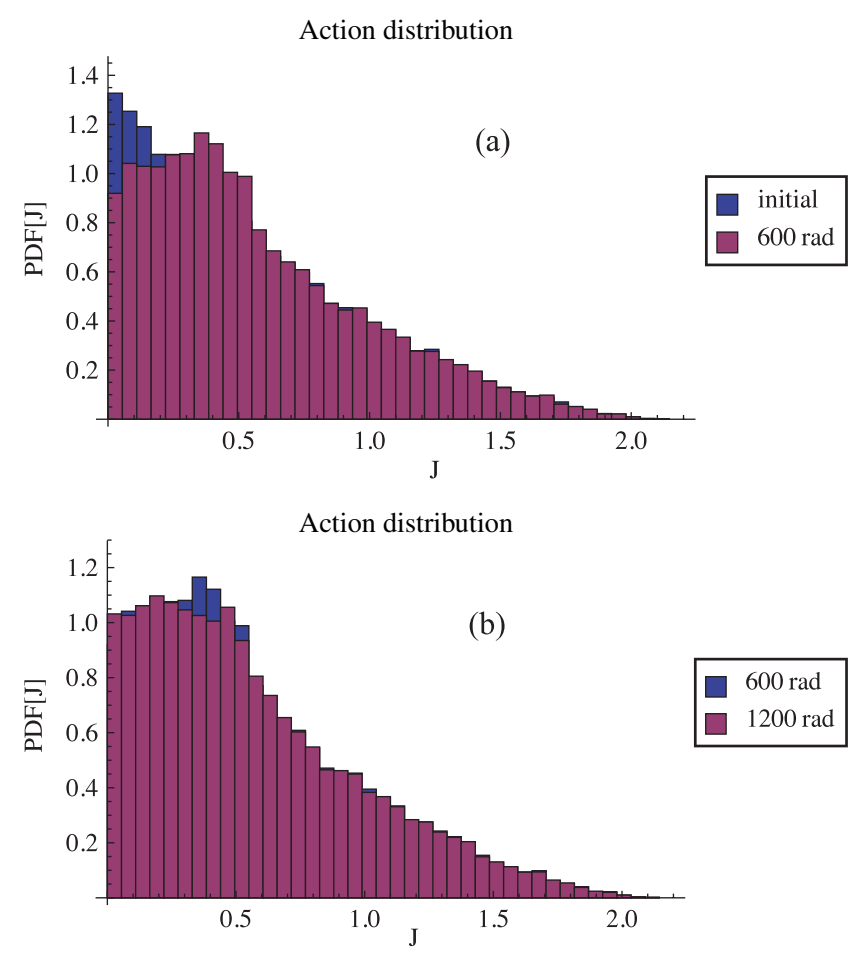

Phase distribution, $\mathrm{t}=0$ \& $\mathrm{t}=\mathrm{T} \operatorname{sim}$

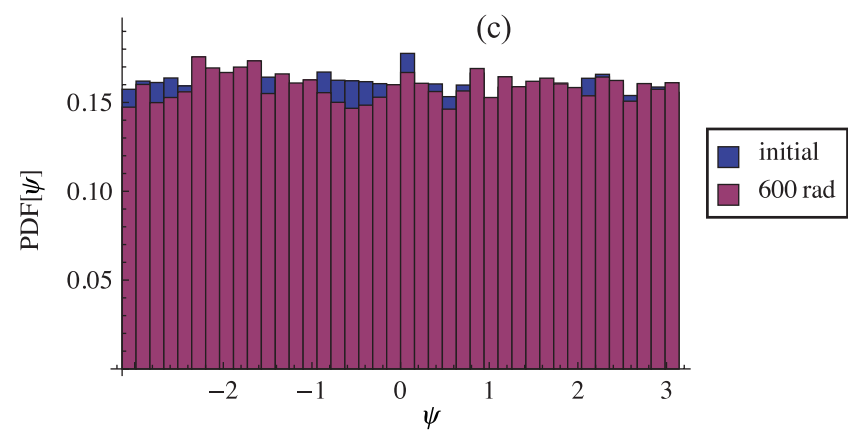

FIG. 1. (a) Distributions over action PDF [J], original (blue) and after the first ramp (pink); the overlapped area is in violet. (b) A similar comparison of the distributions before and after the second ramp. (c) A comparison of the phase distributions PDF $[\psi]$ before and after the first ramp.

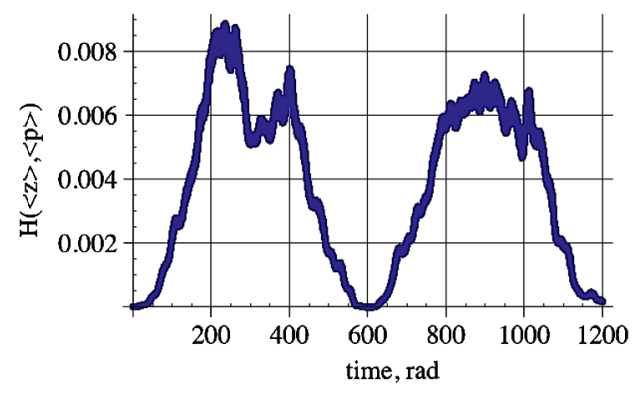

FIG. 2. Time dependence of the unperturbed Hamiltonian taken for the bunch-average coordinate and momentum. pronounced after the second phase modulation cycle. Except for this small difference, the second cycle does not significantly change the distribution. The phase distribution PDF $[\psi]$ after every cycle is as flat as before, showing that no coherent oscillations were excited.

The time dependence of the unperturbed Hamiltonian $H(z, p)=p^{2} / 2+(1-\cos z)$ calculated for the bunchaverage values of the canonical variables $\langle z\rangle$ and $\langle p\rangle$ is shown in Fig. 2. This simulation shows that the adiabaticity of the phase modulation is very important: after every cycle, the Hamiltonian goes to zero. The irregular features of this plot probably reflect the chaotic nature of the anomalous diffusion responsible for the flattening of the distribution.

\section{EXPERIMENT}

The block diagram of the phase modulation hardware used for phase modulating the beam is shown in Fig. 3. A signal generator generates a sine wave where its amplitude and frequency can be programmed and its output is fed into a phase shifter module. The phase shifter modulates the Tevatron low level rf (LLRF) and the result is fed into the Tevatron high level rf (HLRF). Essentially, the components shown in the block diagram produce the following:

$$
f_{\mathrm{HLRF}}=A \sin \left[2 \pi f_{\mathrm{LLRF}} t+\phi_{m}(t) \sin \left(2 \pi f_{m} t\right)+\theta\right],
$$

where $f_{\mathrm{HLRF}}$ is the phase modulated signal sent to the HLRF, $A$ is the amplitude of the signal sent to the HLRF, $f_{\mathrm{LLRF}}$ is the frequency from the LLRF, and $\theta$ is an arbitrary phase. The amplitude $\phi_{m}$ and frequency $f_{m}$ for the phase modulation are set by the signal generator.

The time evolution of the bunch during the experiment is measured using the sampled bunch display (SBD) [20]. Its block diagram is shown in Fig. 3. The SBD measures the bunch profile using a resistive wall current monitor with an oscilloscope that has a $2 \mathrm{GHz}$ bandwidth. The collected data is processed with a LABVIEW program which calculates the following parameters: (i) bunch centroid; (iii) bunch current; and (ii) rms bunch length.

These parameters are then returned to the control system and can be plotted as in Figs. 5 and 9. Furthermore, the snapshots of the bunch from the resistive wall signal can also be downloaded. The SBD trigger has been set up to take five consecutive snapshots of the bunch at $1 \mathrm{~Hz}$. These snapshots are presented in the figures below.

A block diagram of the phase detector used to measure the longitudinal motion of the bunch with respect to the Tevatron $\mathrm{rf}$ is shown in Fig. 3. The $I / Q$ phase detector is a part of the Tevatron longitudinal damper system [4] which essentially takes the sum signal from a stripline pickup, down-converts it with the Tevatron LLRF, and low pass filters it to produce a quadrature signal. The quadrature signal is then measured with a spectrum analyzer. 


\section{Bunch Phase Modulator}

Sine wave source set to synchrotron frequency

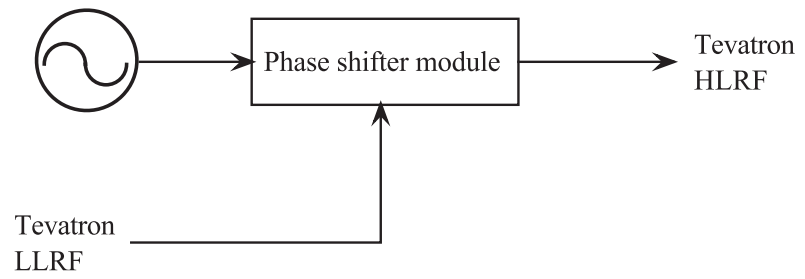

Tevatron Sampled Bunch Display

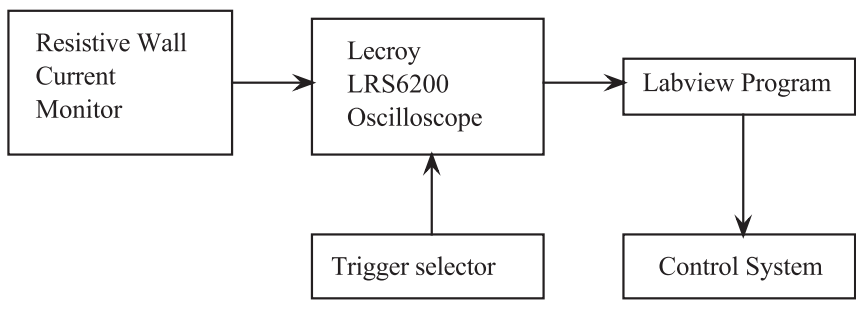

Longitudinal Phase Detector

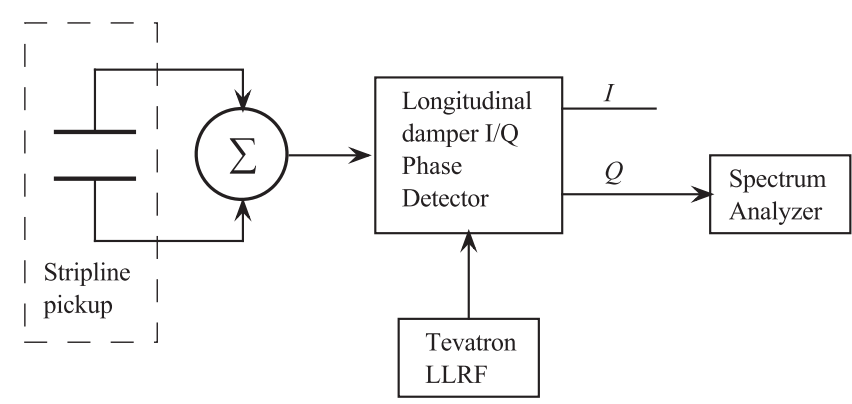

FIG. 3. The block diagrams of the bunch phase modulator and detectors used to monitor the bunches for the described experiments.

The Tevatron parameters relevant to the experiment are shown in Table I. This experiment uses only two coalesced proton bunches and measurements are either taken at the injection energy of $150 \mathrm{GeV}$ or at the flattop energy of $980 \mathrm{GeV}$.

TABLE I. Tevatron parameters relevant to the experiment.

\begin{tabular}{lcc}
\hline \hline Parameter & Value & Units \\
\hline Injection energy & 150 & $\mathrm{GeV}$ \\
Flattop energy & 980 & $\mathrm{GeV}$ \\
Synchrotron frequency at $150 \mathrm{GeV}$ & 87.47 & $\mathrm{~Hz}$ \\
Synchrotron frequency at $980 \mathrm{GeV}$ & 34.75 & $\mathrm{~Hz}$ \\
rf frequency at $150 \mathrm{GeV}$ & 53.103 & $\mathrm{MHz}$ \\
rf frequency at $980 \mathrm{GeV}$ & 53.104 & $\mathrm{MHz}$ \\
Harmonic number & 1113 & \\
Buckets between two injected bunches & 21 & \\
Intensity per bunch & $(200-300) \times 10^{9}$ & \\
\hline \hline
\end{tabular}

\section{A. Results at the injection energy of $150 \mathrm{GeV}$}

The studies presented in this section have been performed at the injection energy of $150 \mathrm{GeV}$. At injection, the bunch nearly fills the bucket and so there are small beam current losses whenever the bunch is modulated. (Results at flattop do not have this problem. See Sec. III B.) In this experiment, $f_{m}$ has been set to $87.47 \mathrm{~Hz}$ because it is the measured synchrotron frequency $f_{s}$ and the bunch is modulated once for $14 \mathrm{~s}$. (Note: theoretically, $f_{m}$ should have been set to a frequency which is smaller than $f_{s}$. However, at the time, this criterion was not appreciated so the experiment was not done.) The phase ramp used in the $150 \mathrm{GeV}$ experiments is not adiabatic and is shown in Fig. 4. The maximum amplitude of the phase modulation has been tested for $\phi_{0}=1,2$, and $3 \mathrm{deg}$, respectively. Experimentally, $\phi_{0}=3 \mathrm{deg}$ has been found to produce the best effect for the duration of the modulation.

Figure 5 shows the modulation duration and the behavior of the bunch current, centroid, and rms bunch length before and after phase modulation. The beam current drops by about $2.3 \%$ and the rms bunch length grows by about $1.8 \%$ after being modulated. The beam current drop is not surprising because the filled bucket is full. The change in
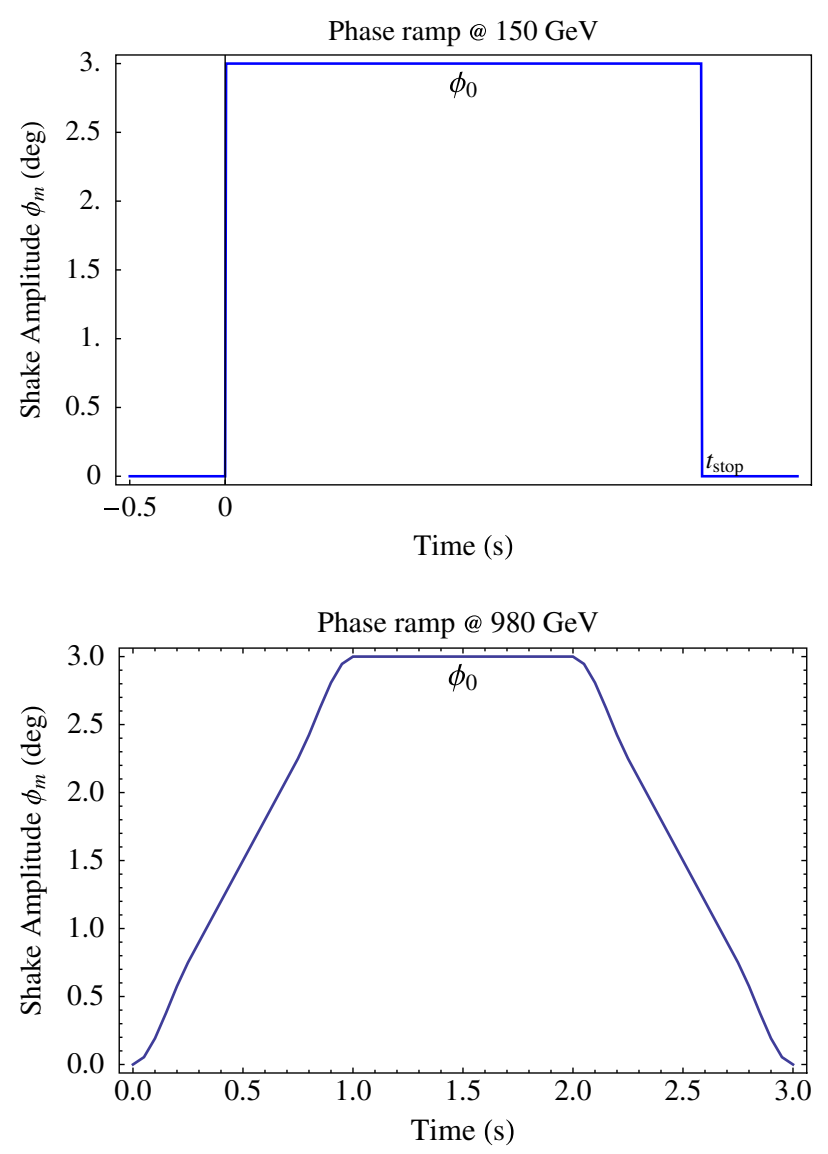

FIG. 4. These are the phase ramps $\phi_{m}(t)$ used at $150 \mathrm{GeV}$ and $980 \mathrm{GeV}$. For the $150 \mathrm{GeV}$ experiments, $t_{\text {stop }}$ is user defined. 


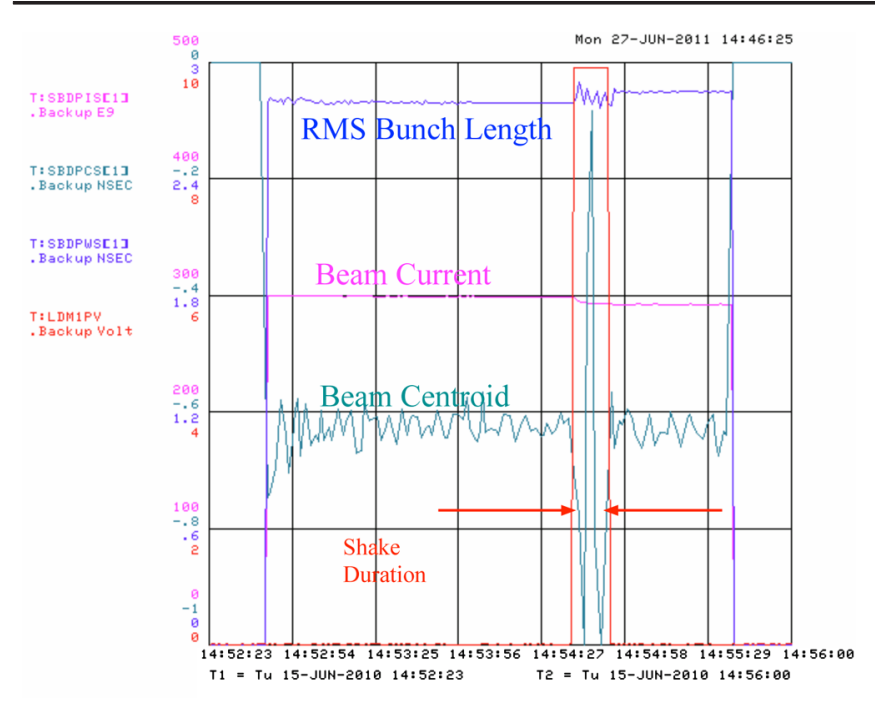

FIG. 5. The data from the SBD system taken at $150 \mathrm{GeV}$ is plotted here: T:SBDPWS, T:SBDPIS, and T:SBDPCS. The beam is modulated for $14 \mathrm{~s}$ and there is some beam loss and bunch length growth. Although the measured bunch centroid looks like it is still oscillating, the snapshots show that the dancing has stopped. See Fig. 6.

rms bunch length is due to the shape change which can be seen clearly in Fig. 6. After the modulation is turned off, a divot structure forms which confirms the prediction previously discussed in Sec. II A.

\section{Contrast to dampers}

The bunch distribution after it has been modulated can be contrasted to the distribution when dampers are used instead to stop the dancing. The before and after distributions are shown in Fig. 7. The effect of dampers on the bunch distribution is to make it more triangular. This can be contrasted to the effect of the modulation technique shown in Fig. 6 where the distribution becomes more rotund. Also, after the dance stops and the dampers are turned off, the bunches do not start dancing again even after the dampers have been off for 5 minutes.

At first glance, the stability of the bunch after the dampers are turned off contradicts the described theory of LLD. Indeed, according to this theory, the LLD threshold is lowered when the distribution function becomes more steep and thus the beam distribution shown in Fig. 7 is less stable after the dampers are turned off than it was before they were turned on. This seemingly contradictory observation can be explained by the extremely small growth rate of the two bunch system. When Landau damping is lost, the growth rate is determined by the coupled bunch wake forces. If these forces are weak enough, the instability takes too long to grow and so it cannot be observed. There are two types of long range wakefields that can be considered as possible candidates for driving the longitudinal coupled bunch instability (LCBI): parasitic cavity modes and the resistive wall wake. Direct
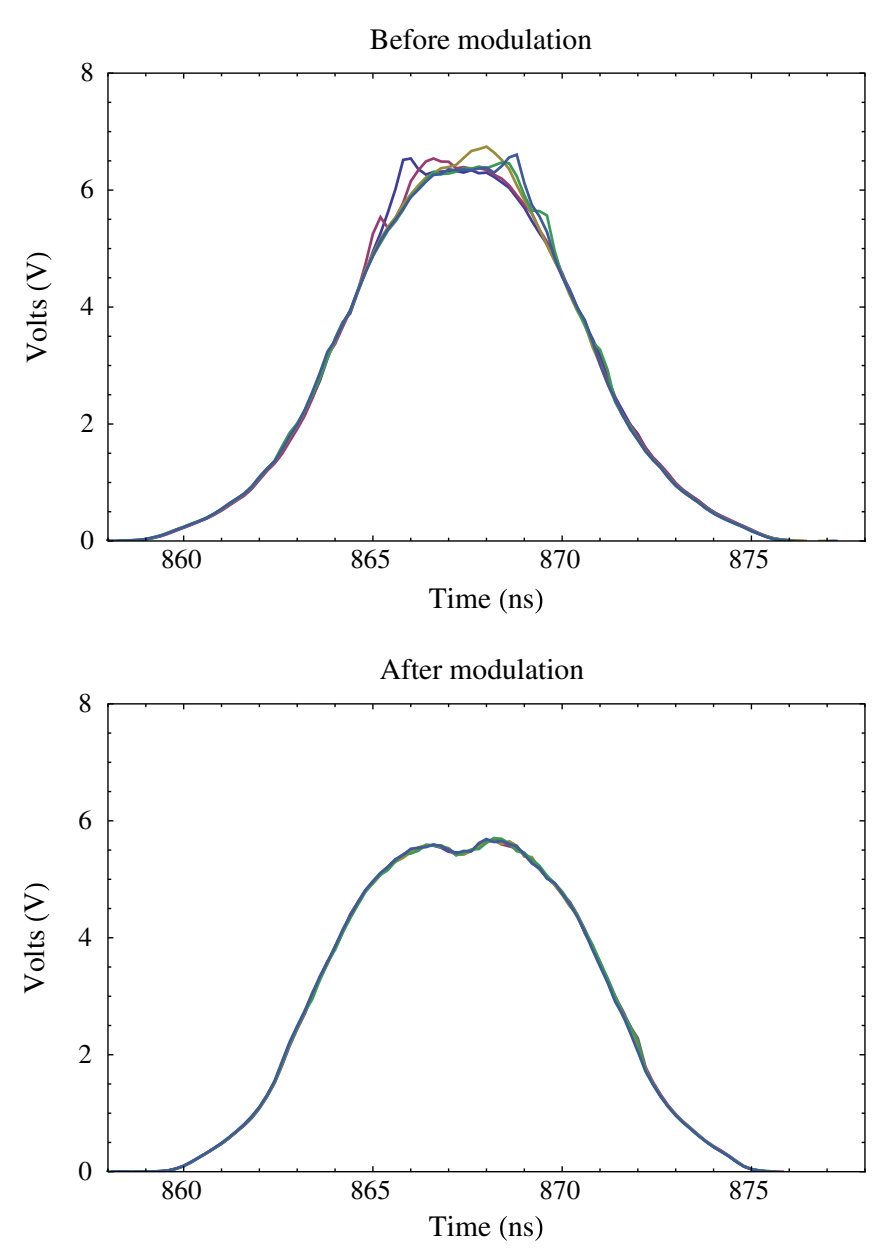

FIG. 6. These are snapshots taken by the SBD before and after modulation at $150 \mathrm{GeV}$. Before any modulation, the bunch is dancing. The result after modulating the beam for $14 \mathrm{~s}$ is the creation of a divot structure in the bunch and stoppage of the tip motion.

calculations show that the resistive wall wake is extremely weak and can be ignored. Even for 36 Tevatron bunches, the calculated resistive wall LCBI growth time is $\sim 10$ days and so the only remaining candidate is the rf cavity modes. According to Ref. [21], the LCBI observations at the top energy for 36 proton bunches can be explained by a parasitic higher order mode at $311 \mathrm{MHz}$ with the caveat that the calculated growth time using the rigid bunch approximation is an order of magnitude faster than the measured one. There are two possible reasons for this discrepancy: the first is a decreased $Q$ value compared to the measured value done in 2000 [21,22] and the second is that the rigid bunch approximation grossly overestimates both the threshold and the growth rate of the instability.

Growth time of the LCBI for the two bunches, $\tau_{2}$, driven by the parasitic cavity mode can be calculated and compared with the growth time $\tau_{M}$ for $M$ equidistant bunches. Using Eqs. (4.123) and (4.128) of Ref. [23], it is straightforward to show that 

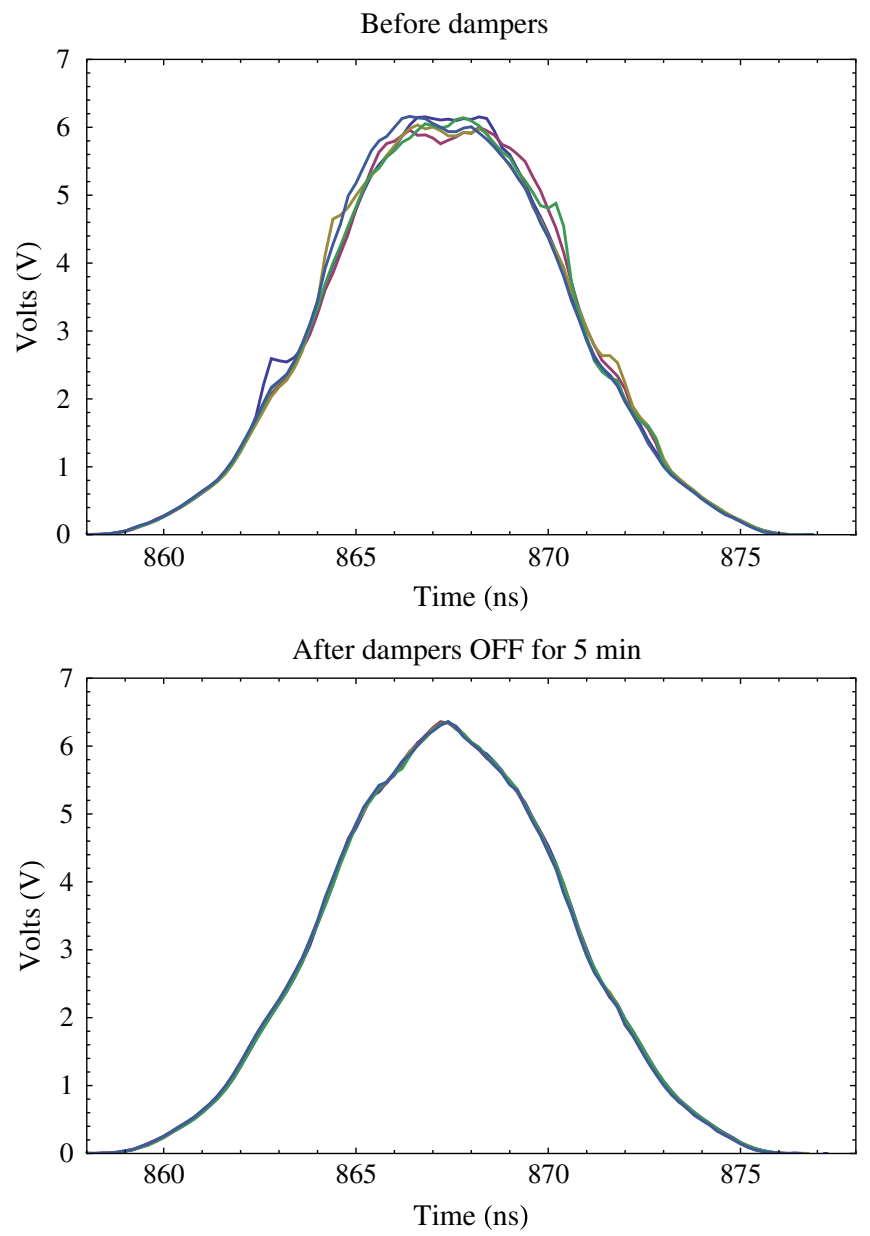

FIG. 7. The before and after effects of using the damper to stop the dance. The distribution after the dampers have stopped the dancing is to make the distribution more triangular in shape.
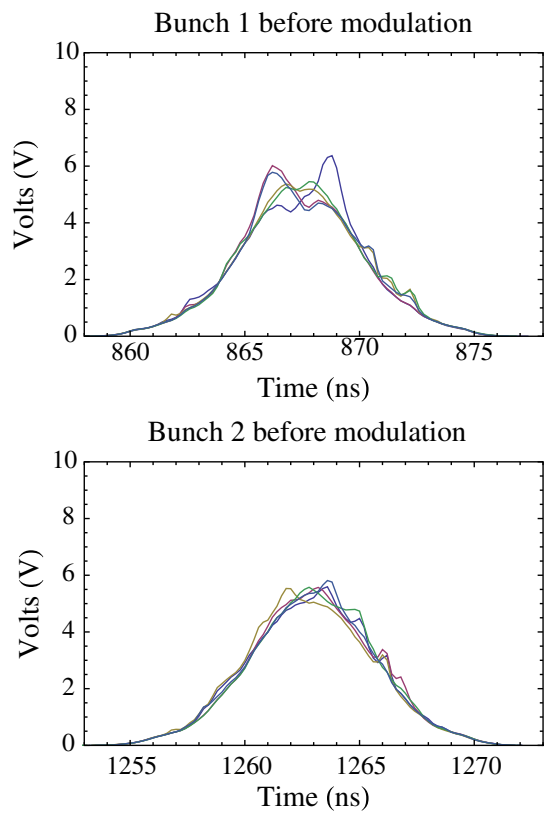

$$
\frac{\tau_{2}}{\tau_{M}} \geq \frac{M}{4 \pi \nu_{s}} \frac{t_{d}}{T_{0}} \exp \left(T_{0} / t_{d}\right)
$$

where $\nu_{S}$ is the synchrotron tune, $T_{0}$ is the revolution time, $\omega_{R}$ is the angular frequency of the parasitic mode, and $t_{d}=$ $2 Q / \omega_{R}$ is its e-fold decay time. This formula was derived under the assumption that the mode decay time $t_{d}$ is shorter than the revolution time and longer than the time separation between the neighboring bunches. This assumption allows the neglect of all multiturn terms in the wake sum of Eq. (4.123) of Ref. [23] for the two bunch case. Application of this formula to the experimental parameters shows that the two bunch growth time is at least $5 \times 10^{3}$ times longer than the standard 36 bunch growth time. According to the measurements of $[21,22]$, the 36 bunch growth time is 2-3 seconds. Thus, Eq. (6) yields at least 3 hours for the growth time for the two bunch system which is much longer than the $\sim 5$ minutes of experimental observations with the dampers off.

\section{Initial bunch shape effects}

The number of modulation cycles required to stop the bunch varied from case to case. Most likely, this is due to the nonoptimized detuning of the modulation frequency and some variations in the bunch intensities and profiles which cause variations in the incoherent tune shifts. Perhaps, a better choice of the detuning parameter $\epsilon=$ $1-f_{m} / f_{s}$ can lead to single modulation damping of the dance, but there was no opportunity to test this.

In this experiment five bunch coalescing is used rather than the usual seven. The initial bunch distribution between bunch 1 and 2 is quite different because the Main Injector
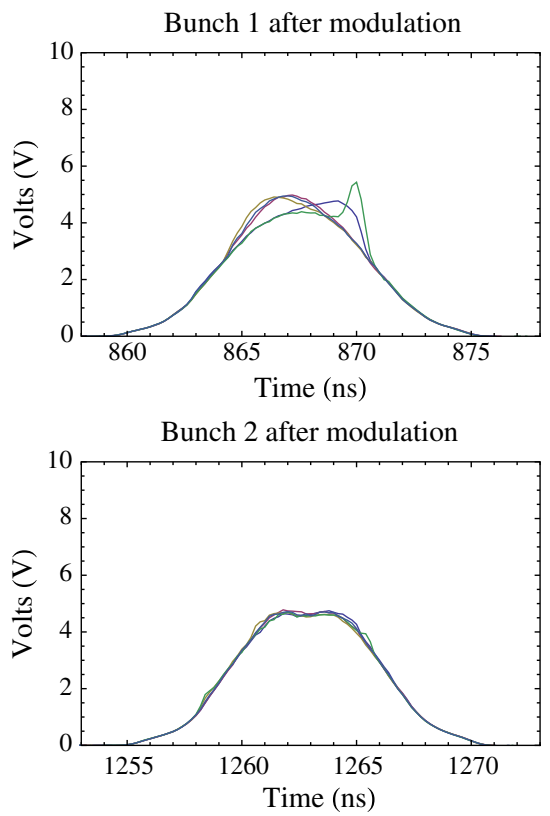

FIG. 8. The initial bunch shape can have an effect on how strongly it must be modulated to stop the dancing. 


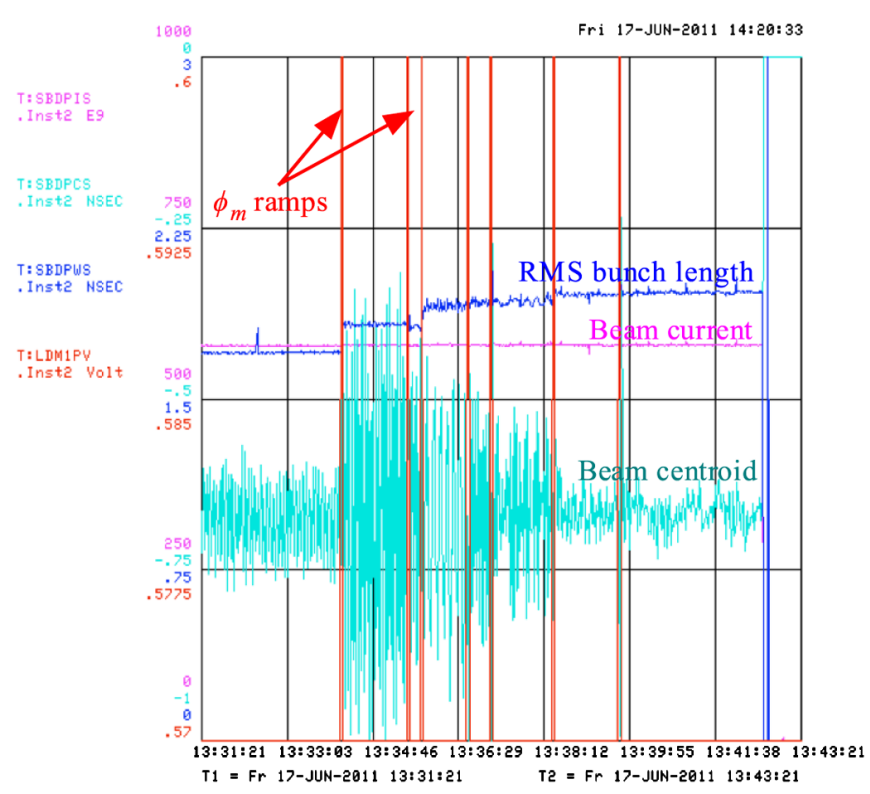

FIG. 9. The beam is modulated 7 times using the $\phi_{m}$ ramp shown in Fig. 4 at $980 \mathrm{GeV}$. After the seventh modulation the dancing stops but there is growth in the rms bunch length because of the shape change.
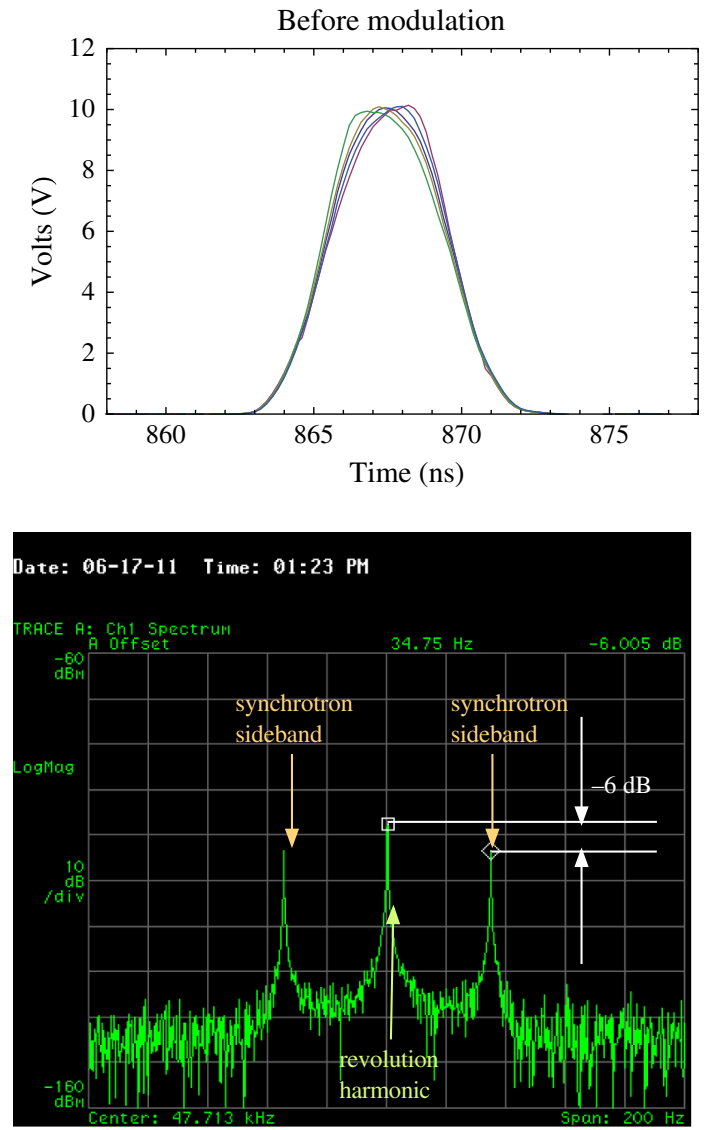

FIG. 10. These figures show the bunch dancing in both the time domain and the frequency domain before any modulating is done at $980 \mathrm{GeV}$. The synchrotron sidebands which are $\pm 34.75 \mathrm{~Hz}$ away from the first revolution harmonic are indicated in the lower picture. has not been tuned up for five bunch coalescing. Therefore, the random effects of untuned coalescing has made bunch 1 dance much more than bunch 2 before the modulation is applied. Figure 8 shows the result of modulating the two bunches at the same time. The bunches are modulated for $7-8 \mathrm{~s}$ at $\phi_{0}=3 \mathrm{deg}$ and the first bunch does not stop dancing while the second bunch stops dancing and gets a divot.

\section{B. Results at the flattop energy of $980 \mathrm{GeV}$}

The bucket is about a factor of 2 larger than the beam size at $980 \mathrm{GeV}$, and thus allows the beam to freely change shape without being constrained by the bucket edges. A $\phi_{m}$
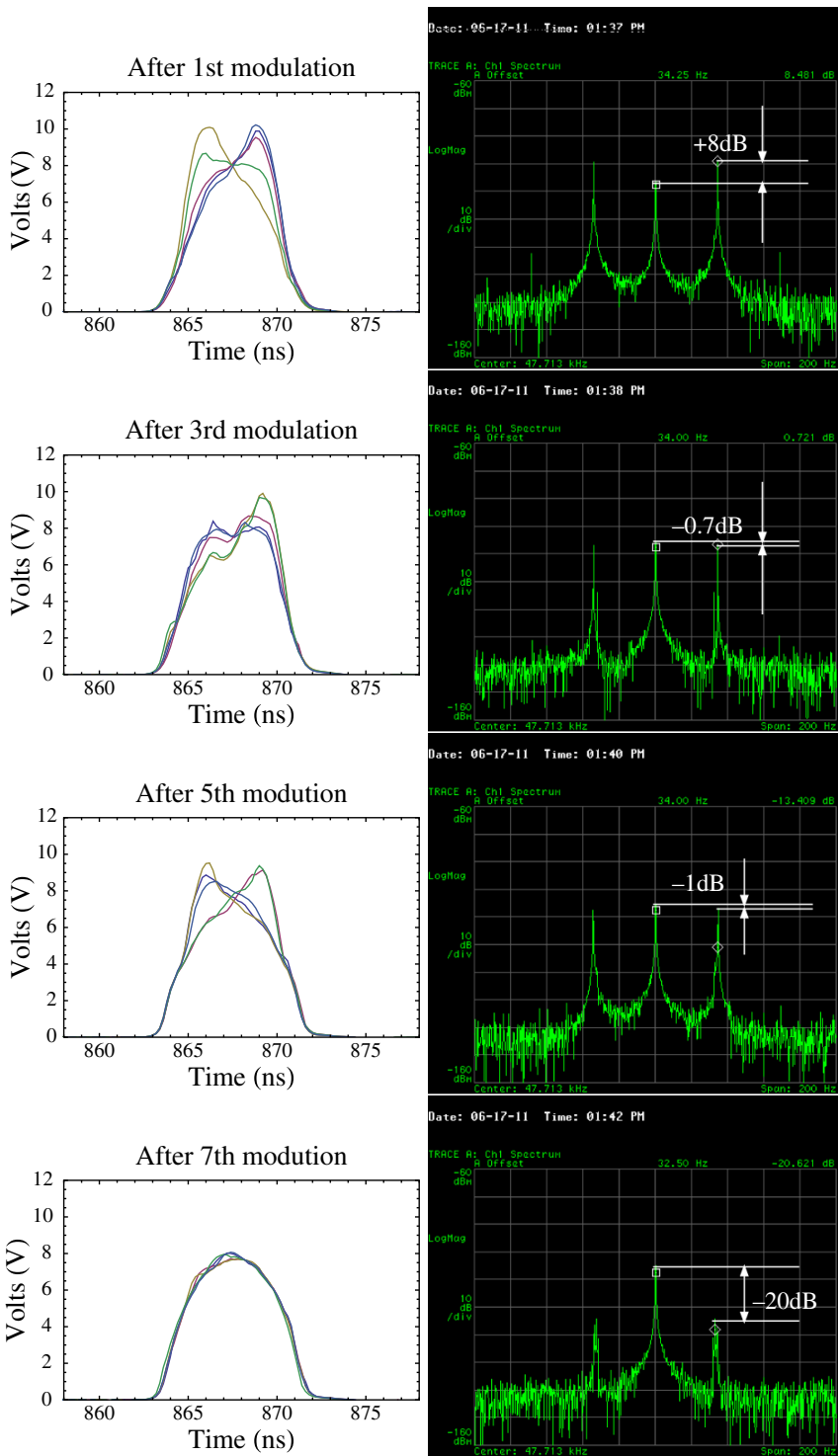

FIG. 11. These figures show how the bunch shape evolves after the first, third, fifth, and seventh modulation. After the seventh modulation, the synchrotron amplitude is reduced by about $14 \mathrm{~dB}$ with respect to its size before any modulation. 
ramp has been created so that there are no abrupt changes in the rf as shown in Fig. 4. Previous experiments have shown that sudden turn-ons can cause some beam loss even though the bucket is large compared to the beam size.

In this experiment, the total phase ramp time is $3 \mathrm{~s}$. The rise and fall time of the ramp has been chosen to be $1 \mathrm{~s}$ because it is slow compared to the synchrotron period of $29 \mathrm{~ms}$. The flattop period can be varied, but for this experiment it has been set to $1 \mathrm{~s}$.

The modulation frequency $f_{m}$ has been set to the measured synchrotron tune $34.75 \mathrm{~Hz}$ and the bunch is modulated 7 times with the $\phi_{m}$ ramp.

Figure 9 shows the seven $\phi_{m}$ ramps and the behavior of the bunch current, centroid, and rms bunch length for the duration of the experiment. The beam current is constant throughout the experiment but the rms bunch length grows by about $18 \%$ (from 1.67 to $1.97 \mathrm{~ns}$ ) by the end of the experiment. It is interesting that the rms bunch length grows after each modulation because of the shape change. A comparison of the bunch shapes before and after the modulation shows that the rms bunch length growth comes from the flatter core of the bunch while its tails remain unchanged.

Figure 10 shows the bunch shape and the spectrum before modulation starts. The spectrum shows the revolution frequency and the synchrotron sidebands which are about $6 \mathrm{~dB}$ smaller than the revolution harmonic. The beam has no quadrupole motion because there are no resonances at twice the synchrotron frequency.

Figure 11 shows both the bunch shape evolution and the spectrum from the phase detector after the first, third, fifth, and seventh modulations. It is clear from these plots that after the first modulation the amplitude of the dance has increased by about $14 \mathrm{~dB}$ relative to the amplitude before modulation. After each subsequent modulation, the amplitude becomes smaller, and after the seventh modulation, the dance amplitude has decreased by $14 \mathrm{~dB}$ relative to the

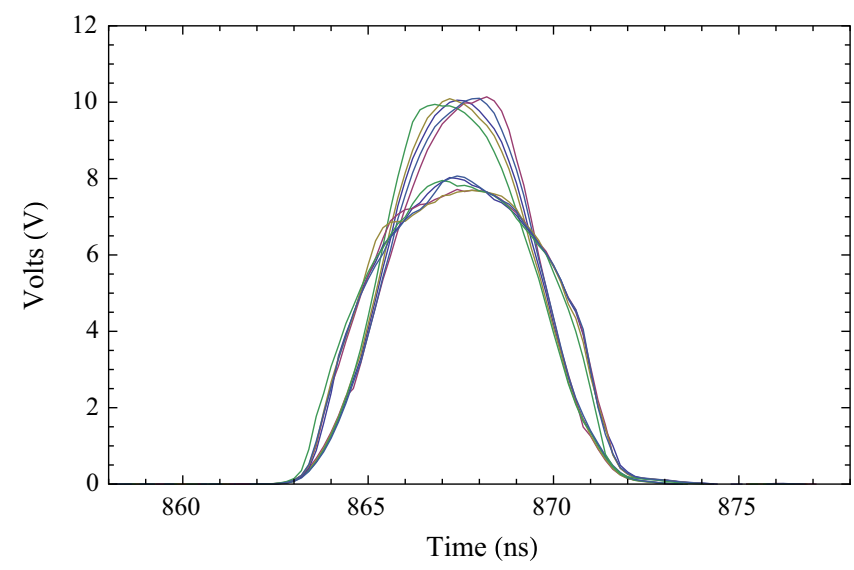

FIG. 12. All the traces which are collected before and after the seventh modulation are plotted together here. This clearly shows the shape change at the end of the experiment. amplitude before the first modulation. The shape of the bunch after the seventh modulation has clearly changed. Figure 12 shows superimposed snapshots of the bunch before and after the seventh modulation.

\section{CONCLUSION}

Similar ideas for bunch distribution flattening have been suggested and implemented in the KEK-PS [24,25] and the KEK Photon Factory [26]. This technique is also routinely applied in the CERN SPS to blow up the longitudinal emittance for stabilizing the beam [27]. However, in all of these cases, narrow band rf noise around the synchrotron sidebands is used as the excitation. In the KEK-PS and SPS, the rf perturbation is applied to the voltage amplitude while at the KEK Photon Factory, noise is applied to the rf phase. The experiments described in this paper take a different approach: instead of noise, the rf phase is excited at the synchrotron frequency, and its amplitude is ramped adiabatically. This technique works because anomalous diffusion flattens the bunch distribution. It is also possible that this technique is able to finely regulate the width where the distribution is flattened while keeping the remaining distribution untouched.

As was previously discussed in Sec. II, this method of bunch flattening is very sensitive to the detuning of the modulation frequency from the synchrotron frequency. In all these experiments, the rf phase was modulated at the measured coherent synchrotron frequency which can be higher or lower than the proper value calculated with the potential well distortions taken into account. In the experiments, several consecutive rf modulation cycles are needed to stop the bunch oscillations, while theoretically, with the proper detuning of the phase modulation frequency, only one modulation cycle should be able to stop it. Presently, there is no convincing answer for the number of rf modulation cycles needed in these experiments. However, part of the answer has to lie in controlling the detuning of the phase modulation frequency. Unfortunately, due to the lack of machine studies time and the shutdown of the Tevatron [28], the effects of detuning have not been explored. Hopefully, future studies in other machines will shed light on this issue.

All of the Tevatron experiments discussed here show that an rf phase modulation that is ramped to an amplitude of a few degrees for a duration of a few seconds can flatten the low amplitude distribution of the beam. In some cases, a divot forms à la computer simulations. These beam studies show that stabilization does happen as soon as the bunch is flattened, confirming the proposal that resonant $\mathrm{rf}$ modulation can stop the beam from dancing.

\section{ACKNOWLEDGMENTS}

The authors wish to thank V. Lebedev for discussions and R. Madrak for reading and correcting errors in 
this manuscript. This work is supported by Fermi Research Alliance, LLC under Contract No. DE-AC0207CH11359 with the United States Department of Energy.

[1] One coalesced bunch means that 7 bunches from the Main Injector are coalesced into one high current bunch and injected into the Tevatron. For our experiment, two coalesced bunches separated by 21 buckets are used.

[2] W. Guo et al., FNAL Technical Document No. TM-2203, 2003.

[3] R. Moore et al., in Proceedings of the 20th Particle Accelerator Conference, Portland, OR, 2003, edited by J. Chew et al. (IEEE, New York, 2003), pp. 1751-1753.

[4] C. Y. Tan and J. Steimel, in Proceedings of the 20th Particle Accelerator Conference, Portland, OR, 2003 (Ref. [3]), pp. 3071-3073.

[5] A. Burov, in Proceedings of the 2011 Particle Accelerator Conference, NY, USA (IEEE, New York, 2011), pp. 94-96.

[6] A. Burov, Fermilab-pub-11-174-AD [http://lss.fnal.gov/ archive/2011/pub/fermilab-pub-11-174-ad.pdf].

[7] A. Burov, FNAL AD Document No. BD3618, 2010.

[8] M. Henon, Astron. Astrophys. 114, 211 (1982).

[9] N. G. van Kampen, Physica 23, 641 (1957).

[10] G. Ecker, Theory of Fully Ionized Plasmas (Academic Press, New York, 1972).

[11] F. Sacherer, Report No. CERN/SI-BR/72-5, 1972.

[12] G. Besnier, Nucl. Instrum. Methods 164, 235 (1979).

[13] A. Hofmann and F. Pedersen, IEEE Trans. Nucl. Sci. 26, 3526 (1979).
[14] S. Y. Lee and K Y. Ng, in Proceedings of HB2010, Morschach, Switzerland (2010), pp. 639-643 [http:// accelconf.web.cern.ch/AccelConf/HB2010/index.htm].

[15] E. Metral, CERN-AB-2004-002 (ABP), 2004.

[16] I. S. Gonzalez and F. Zimmermann, in Proceedings of the 11th European Particle Accelerator Conference, Genoa, 2008 (EPS-AG, Genoa, Italy, 2008), pp. 1721-1723.

[17] D. Jeon et al., Phys. Rev. Lett. 80, 2314 (1998).

[18] H. Huang et al., Phys. Rev. E 48, 4678 (1993).

[19] S. Y. Lee, Accelerator Physics (World Scientific, Singapore, 2004).

[20] S. Pordes et al., in Proceedings of the 21st Particle Accelerator Conference, Knoxville, edited by C. Horak (IEEE, Piscataway, NJ, 2005), pp. 1362-1365.

[21] C. Y. Tan, FNAL AD Document No. BD1597, 2005.

[22] C. Y. Tan, Higher order mode measurements were performed by the author, 2000.

[23] A. Chao, Physics of Collective Beam Instabilities in High Energy Accelerators (J. Wiley \& Sons, Inc., New York, 1993).

[24] T. Toyama, Nucl. Instrum. Methods Phys. Res., Sect. A 447, 317 (2000).

[25] T. Toyama et al., in Proceedings of the European Particle Accelerator Conference, Vienna, 2000 (EPS, Geneva, 2000), pp. 1578-1580.

[26] S. Sakanaka et al., Phys. Rev. ST Accel. Beams 3, 050701 (2000).

[27] P. Baudrenghein et al., in Proceedings of the 20th Particle Accelerator Conference, Portland, OR, 2003 (Ref. [3]), pp. 3050-3052.

[28] The Tevatron was retired from service in 2011. 